\title{
ARTICLE
}

\section{Development of a new calculation system to estimate decontamination effects}

\author{
Tomoharu Hashimoto*, Masahiro Kondo, Hideho Gamo, Ryuichi Tayama and Toshihisa Tsukiyama
}

Hitachi-GE Nuclear Energy, Ltd., 3-1-1, Saiwai-cho, Hitachi-shi, 317-0073, Japan

\begin{abstract}
The Japanese government plans to conduct decontamination tasks in radioactively contaminated areas. For such a situation, we developed a system that evaluates radiation dose rates in a wide radioactively contaminated area by utilizing our radiation dose evaluation technology. We also applied this system to actual decontamination model project and confirmed that this system was capable of calculating predicted dose rate after decontamination with sufficient accuracy.
\end{abstract}

Keywords: decontamination; Fukushima; contour map; SOPHIDA; MCNP5; Monte Carlo; CDE; PHITS

\section{Introduction}

In order to cope with the environmental contamination caused by radioactive substances leaked out from the reactors at the Fukushima Daiichi nuclear power plant, several decontamination tasks conducted. To promote these tasks effectively, a calculation system to evaluate radiation dose must have following functions.

- Obtaining highly accurate results in a short time

- Evaluating the dose-reduction effect of decontamination

- Rationalizing entry work for calculation input

- Generating radiation dose contour maps to easily confirm levels of environmental contamination

On the other hand, the following problems arise when applying conventional calculation code for shield design to decontamination tasks.

- Calculation dealing with many kinds of radioactive nuclides strength at one time is difficult.

- In order to confirm radiation dose rate-reduction effects, the conditions of radioactivity are changed many times. Therefore, these procedures take much time.

- A sufficient number of workers well versed in radiation dose calculation cannot be secured.

- Checking input entails a heavy workload.

- Calculation takes a long time.

- Functions to generate radiation dose contour maps are lacking.

In order to address the problems above, we have developed a new simulation system called- "SOPHIDA (Source Of Photons and Integrated Dose Analysis)" for evaluating radiation dose rates in a wide radioactively contaminated area. This system can be used in the Windows environment, calculate the evaluation result in a several tens of minutes.

This paper describes the development specifications, overview of the system, and application to actual decontamination.

\section{Development specification}

As the first step of development, we placed top priority on calculation speed and on reducing the workload. Table 1 lists the development specifications.

Table 1. The Development specification.

\begin{tabular}{|l|l|l|}
\hline \multicolumn{1}{|c|}{ Item } & \multicolumn{1}{|c|}{ Specification } & \multicolumn{1}{c|}{ Remarks } \\
\hline Geometry & 3-dimensional & \\
\hline Shapes of source & $\begin{array}{l}10 \mathrm{~m} \times 10 \mathrm{~m} \\
100 \mathrm{~m} \times 100 \mathrm{~m}\end{array}$ & \\
\hline Radioactive nuclide & \multicolumn{1}{|c|}{${ }^{134} \mathrm{Cs},{ }^{137} \mathrm{Cs}$} & $\begin{array}{l}\text { A ratio is } \\
\text { arbitrary }\end{array}$ \\
\hline Calculation range & $2000 \mathrm{~m} \times 2000 \mathrm{~m}$ & One case \\
\hline Shield & $\cdot$ Soil & $\cdot 1.8 \mathrm{~g} / \mathrm{cm}^{3}$ \\
& $\cdot$ Concrete & $\cdot 2.15 \mathrm{~g} / \mathrm{cm}^{3}$ \\
& $\cdot$ Iron & $\cdot 7.8 \mathrm{~g} / \mathrm{cm}^{3}$ \\
\hline Skyshine & Calculate & $20,40,60,80,90$ \\
& Conical Beam \\
& Response & \\
& Function $)^{[1]}$ & \\
\hline Accuracy & MCNP $\pm 20 \%$ & \\
\hline
\end{tabular}

*Corresponding author. Email:Tomoharu.Hashimoto.uq@hitachi.com 


\section{System overview}

\subsection{Principle of calculation}

Because radiation dose rate is measured as the typical radiation dose rate from a surface of $10-\mathrm{m}$ square or 100-m square, calculation area is divided into a lot of meshes by $10-\mathrm{m}$ square or $100-\mathrm{m}$ square. In addition, it is possible to define another radiation source condition to each mesh. Furthermore, an evaluating point is also distributed into calculation area at mesh state. The radiation dose rate calculation is carried out each other from all of radiation sources to all of evaluating points. The contribution from all the radiation sources is totaled at an evaluating point. Finally, a dose rate contour map can be created by using all the calculation results of the evaluating point which was distributed over calculation area.

In order to simplify, a principle is explained in detail using one of the divided source. When the dose rate measured in a radioactive contaminated area is entered into the SOPHIDA system, it will change the ambient dose equivalent $\mathrm{H}^{*}(10)$ to contamination density as the radiation source by exclusive coefficient. In the next process, radiation dose rate at an evaluating point can calculate based on contamination density. The dose rate of an evaluating point is calculated in consideration of the radiation attenuation by the distance and by shielding. These calculations are carried out by approximation formula which is defined by MCNP5 calculation result. Figure 1 shows the principle of calculation.

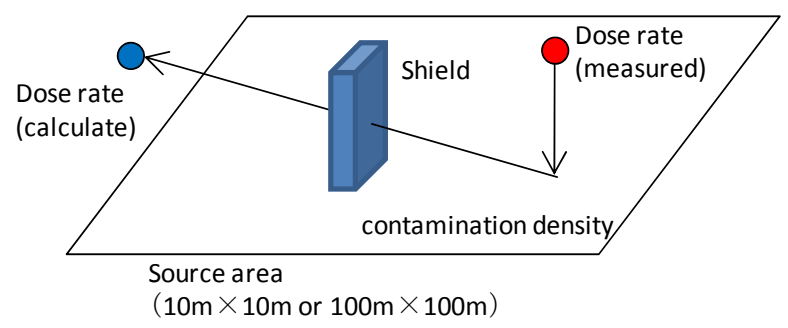

Figure 1. Principle of calculation.

\subsection{Approximation formula}

In order to reduce the calculation time, we defined the empirical formula proposed by Harima[1], and it was adopted in the SOPHIDA calculation system. This formula is shown below.

$$
F(t)=A e^{(-B-C t)} t^{(-D-E t)}
$$

where

$\mathrm{F}(\mathrm{t})$ is dose rate at the evaluating point.

$\mathrm{t}$ is distance from the radiation source to the evaluating point.

A, B, C, D, and E are fitting parameters.

Each fitting parameter of a formula is given from a MCNP5 calculation result according to radiation source condition. This formula is in agreement with the calculation results of MCNP5 within $\pm 20 \%$. This formula can calculate the relation between distance and the radiation dose rate. Figure 2 show the attenuation curves calculated by approximation formulas adopted in the SOPHIDA.

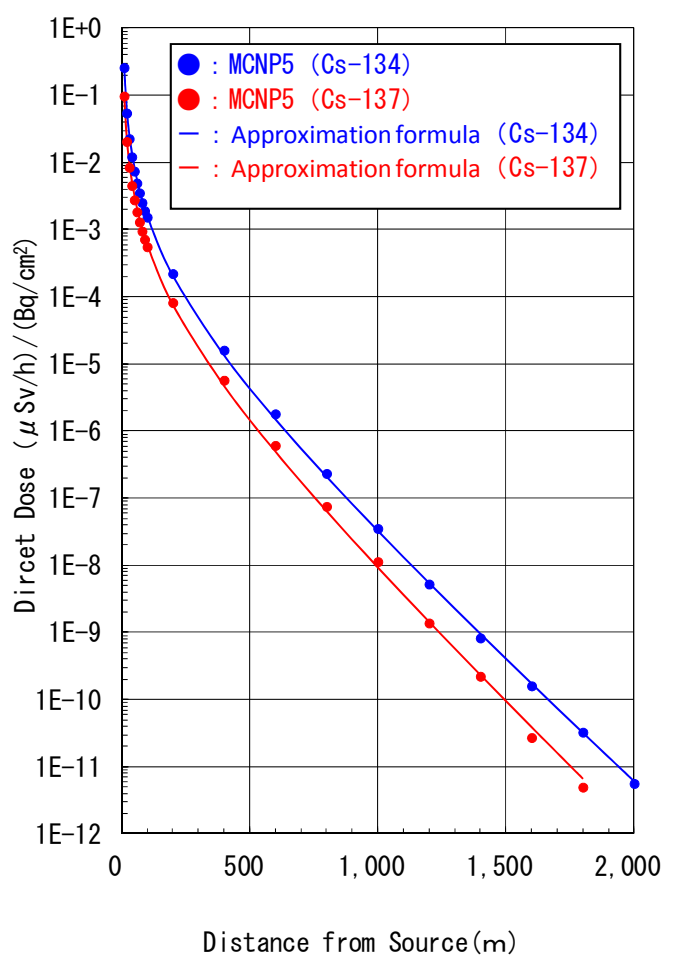

Figure 2. Attenuation curves calculated by approximation formulas for ${ }^{134} \mathrm{Cs},{ }^{137} \mathrm{Cs}$ (10-m square source).

Since the attenuation ratio by shield given from another MCNP5 calculation result is included in the system, SOPHIDA can take a shielding effect into consideration. The soil, concrete, and iron can be chosen as a shield. When there are shields around the calculation area, Sky-shine dose rate is taken into account in dose rate calculation based on Conical Beam Response Function. Figure 3 show the attenuation ratio of shields given from MCNP5.

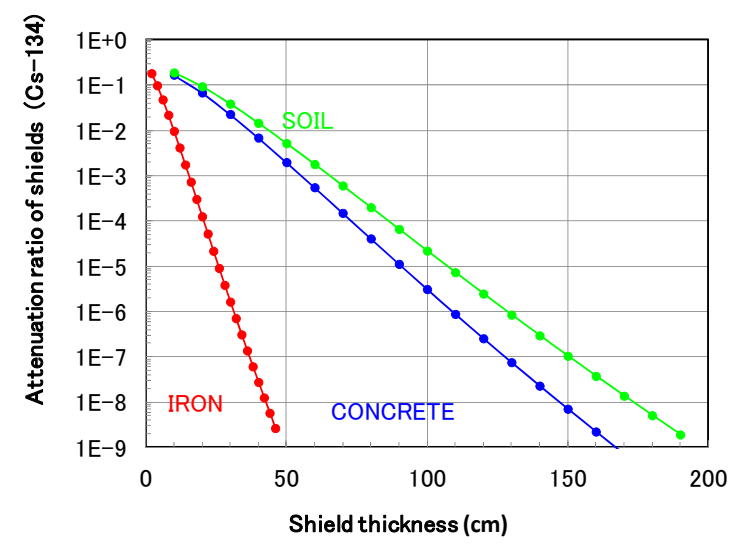

Figure 3. The attenuation ratio of shields $\left({ }^{134} \mathrm{Cs}\right)$. 
In addition, the verification of this system had been already performed [2] by the comparison with CDE [3] or PHITS code [4]. As a result of verification, the difference between this system and the above codes has been confirmed to be within $\pm 20 \%$.

\section{Application to actual decontamination}

\subsection{Application to the decontamination model project}

JAEA conducted "The decontamination model project". This project was carried out to verify the following before conducting full-scale decontamination,

- The effects of various decontamination technologies

- How to plan for proceeding with decontamination work efficiently

As the SOPHIDA can provide useful information for planning decontamination, we applied the SOPHIDA system to this project. We were also able to confirm the following points as by-products:

- Validity of the calculation result

- Accuracy of predicted dose after decontamination by calculation

Table 2 lists the municipalities for which dose rates after decontamination were predicted using SOPHIDA. Figure 4 shows a map of the area around the Fukushima Daiichi nuclear power plant and the location of the area targeted for decontamination.

Table 2. Applicable area.

\begin{tabular}{|l|c|l|}
\hline \multicolumn{1}{|c|}{ Municipality } & $\begin{array}{c}\text { Distance from } \\
\text { nuclear plant }\end{array}$ & \multicolumn{1}{|c|}{ Remarks } \\
\hline Okuma town(1) & $5 \mathrm{~km}$ & \\
\hline Okuma town(2) & $0.5 \mathrm{~km}$ & $\begin{array}{l}\text { Adjacent to } \\
\text { nuclear power } \\
\text { plant. }\end{array}$ \\
\hline Kawauchi village & $15 \mathrm{~km}$ & \\
\hline Naraha town(1) & $15 \mathrm{~km}$ & \\
\hline Naraha town(2) & $20 \mathrm{~km}$ & \\
\hline Hirono town & $25 \mathrm{~km}$ & \\
\hline
\end{tabular}

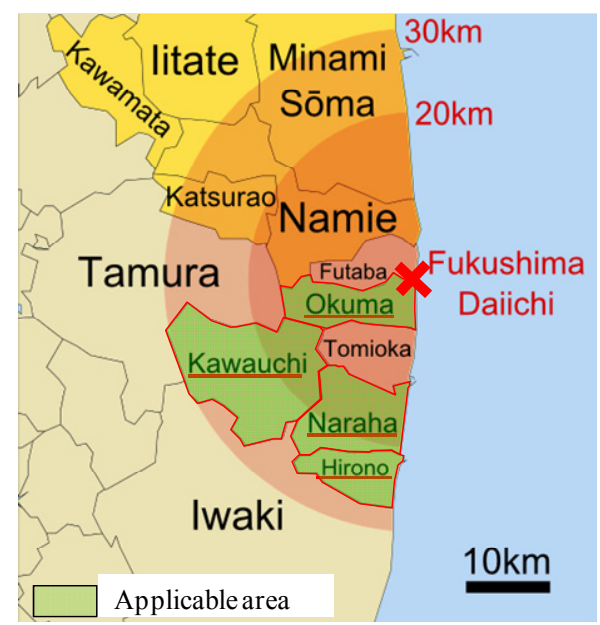

Figure 4. Map of area around the Fukushima Daiichi plant.

\subsection{Comparison with the measurement result}

SOPHIDA was first applied to Okuma town (1) . By comparing the measured values with the results calculated by SOPHIDA in this area, we have confirmed the validity of the calculated results. The Validity was evaluated by using the $\mathrm{C} / \mathrm{E}$ value. As a result, the $\mathrm{C} / \mathrm{E}$ value in Okuma town (1) was 0.8 on average. Figure 5 shows an aerial photo of Okuma town (1). Figure 6 shows an air dose rate map before decontamination in this area as calculated by SOPHIDA based on dose rate measurement results for the ground surface. And Figure 7 shows the $\mathrm{C} / \mathrm{E}$ values that indicate the ratio of measured and calculated air doses.

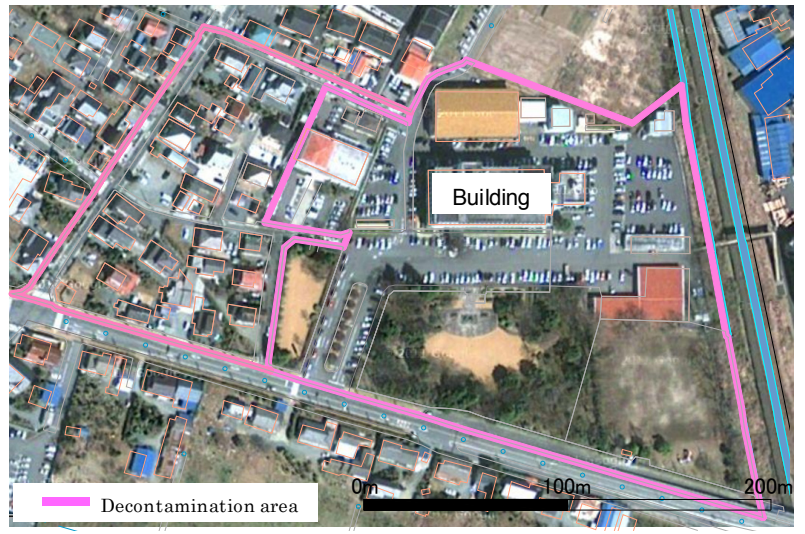

Figure 5. Aerial photo of Okuma town (1).

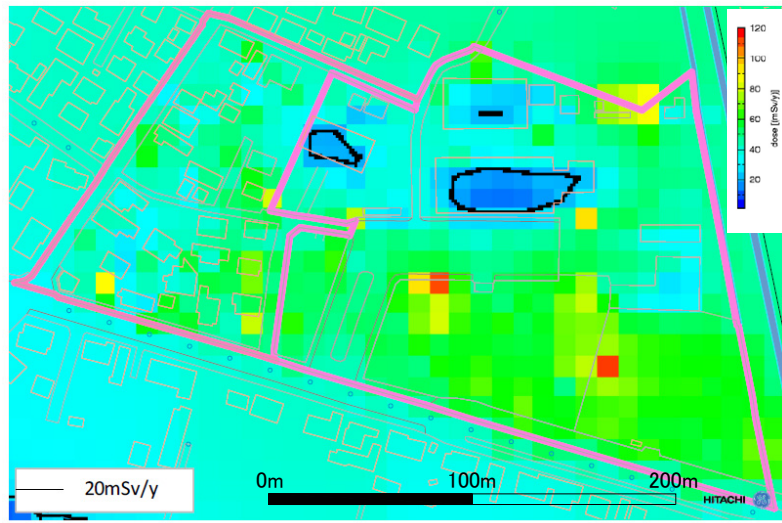

Figure 6. Air dose rate map before decontamination [5].

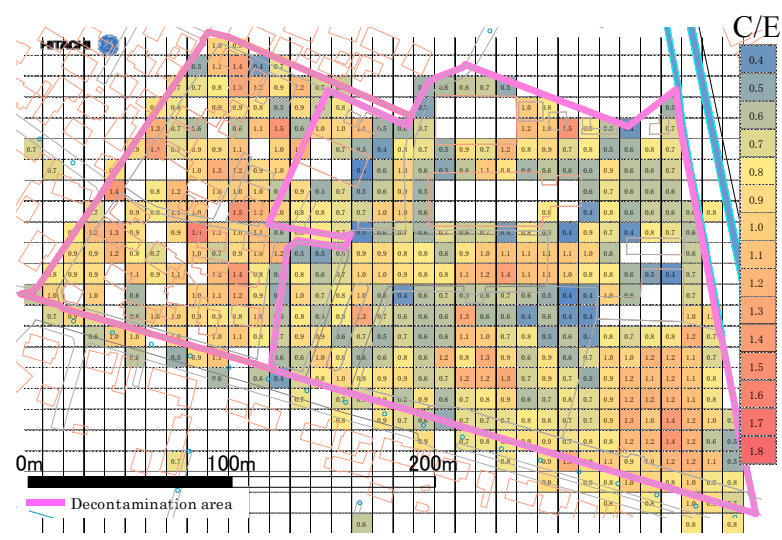

Figure 7. C/E values (for Okuma town (1)). 
As there are many trees in this area, the calculation results tend to become slightly underestimated. This can be explained by the fact below in confirmation by comparing Figures 5 and 7 . The range of vegetation such as trees almost agrees with the low value range of $\mathrm{C} / \mathrm{E}$. If the radiation source on the tree is entered as additional radiation sources, this underestimation would be improved. By improving the evaluation results as described above, SOPHIDA would output the appropriate results.

\subsection{Confirmation of the predicted dose map}

The radiation dose rate map of Okuma town (2) was created using the same evaluation method used for Okuma town (1). Moreover, the predicted dose rate map after decontamination of Okuma town (2) was calculated by entering the decontamination factors according to the decontamination plan. Finally, the actual dose rate map after decontamination was compared with the predicted map. Table 3 lists the decontamination factors (DF) used in calculation. Figure 8 shows an aerial photo of Okuma town (2). Figure 9 shows the actual dose rate map before the decontamination of Okuma town (2). Figure 10 shows the predicted dose rate map after decontamination of Okuma town (2). Figure 11 shows the actual dose rate map after decontamination of Okuma town (2).

According to the result of comparing Figures 10 and 11 , it was confirmed that SOPHIDA can calculate the predicted dose rate after decontamination with sufficient accuracy.

Table 3. Decontamination factors using in calculation.

\begin{tabular}{|l|c|l|}
\hline \multicolumn{1}{|c|}{ Contents } & DF & \multicolumn{1}{|c|}{ Area of application } \\
\hline Topsoil removal & 10 & $\begin{array}{l}\text { Residential area, } \\
\text { Fields, Unpaved road }\end{array}$ \\
\hline Surface stripping & 5 & Paved road \\
\hline Removing humus layer & 3 & Forest (Conifer) \\
\hline Removing humus layer & 2 & Forest (Non-conifer) \\
\hline
\end{tabular}

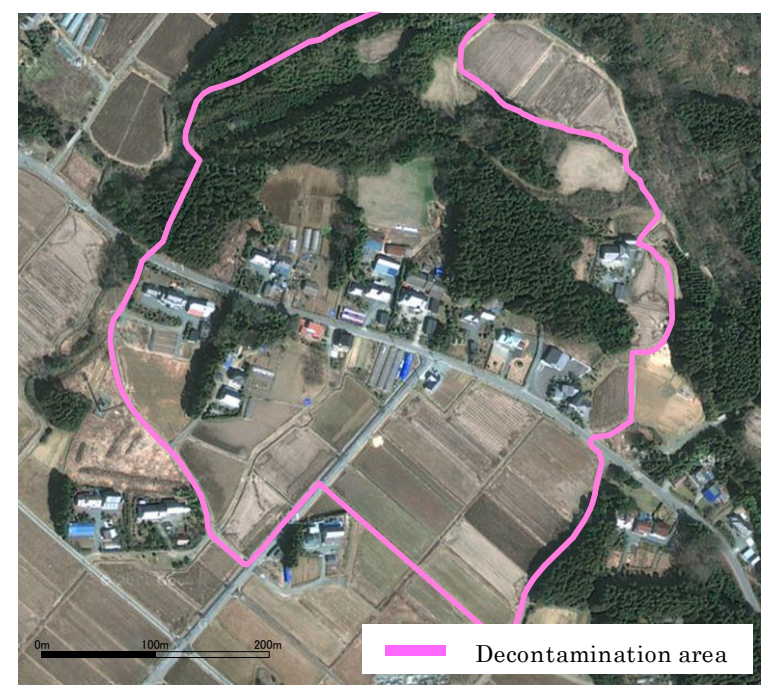

Figure 8. Aerial photo of Okuma town (2).

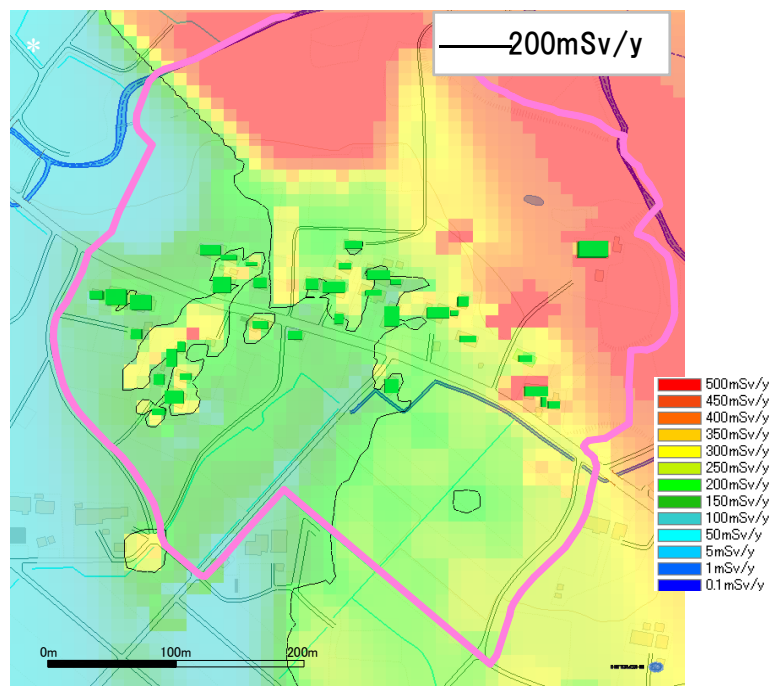

Figure 9. Actual dose rate map before decontamination of Okuma town (2) [5].

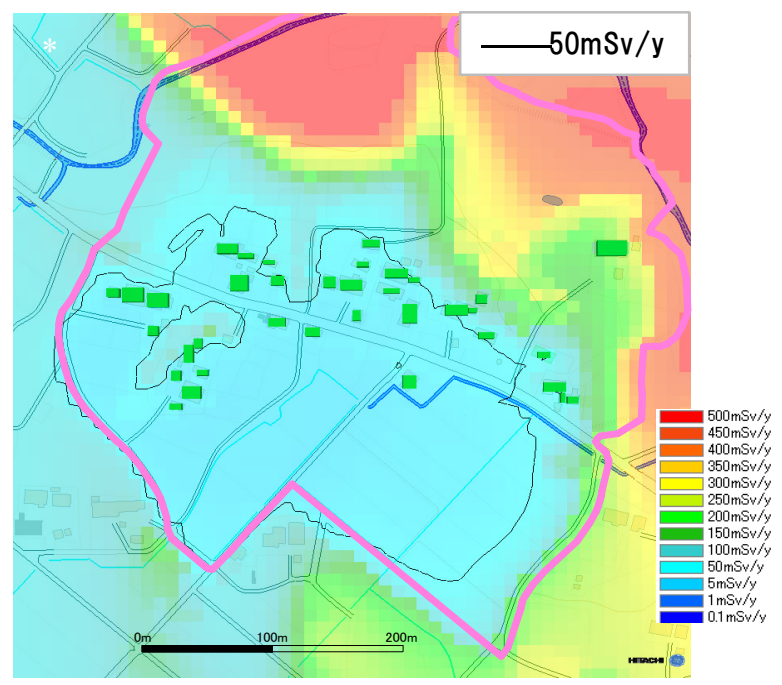

Figure 10. Predicted dose rate map after decontamination of Okuma town (2) [5].

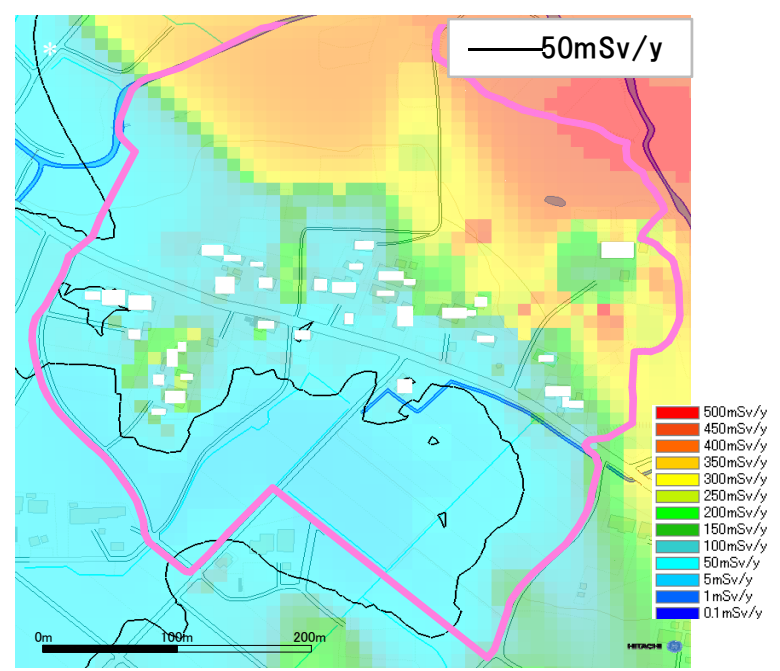

Figure 11. Actual dose rate map after decontamination of Okuma town (2) [5]. 


\subsection{Consideration for the decontamination model project}

Because SOPHIDA was applied to the decontamination project, we were able to know about the actual state of contamination. Based on the experience gained in this project, we got the following consideration using SOPHIDA.

(1) Radiation influence from outside of decontamination range

In the decontamination model project, because the decontamination range was limited to a radius of about $200 \mathrm{~m}$, the large radiation contribution from non-range decontamination was still remaining. Therefore, in order to obtain a sufficient dose reduction effect, additional decontamination would be required until $100 \mathrm{~m}$ outside from the planning range.

(2) Influence other than radiation source located on the ground.

In the decontamination model project, because the surface contamination on the ground had been intensively decontaminated, the contribution from the remaining radiation sources (mainly on trees) other than the ground surface could not be ignored. Therefore, we considered that the decontamination of the radiation source on the tree concurrently with radiation source on the ground is necessary and effective.

\section{Conclusion}

We developed the SOPHIDA system for decontamination tasks. This system enables rapid, rational, easy calculation by non-expert calculation workers. And it can display a radiation dose rate contour map for effective support. Such a map can be used effectively for planning to reduce public exposure to radiation.

From now on, SOPHIDA can be immediately applied to actual decontamination tasks, and additional functional improvement would be carried out periodically if needed.

\section{References}

[1]Y. Harima, H. Hirayama, Y. Sakamoto, N. Sasamoto, F. Masukawa, H. Nakashima, K. Hayashi, H. Handa, R. Tayama, N. Kurosawa, M. Nemoto and T. Abe, Validity of the four-parameter empirical formula in approximating the response functions for gamma-ray, neutron, and secondary gamma-ray skyshine analysis, Journal of Nuclear Science and Technology, Vol.40, No. 8, (2003), pp.569-578.

[2] T. Hashimoto, M. Kondo, R. Tayama and H. Gamo, Development of new simulation software system to evaluate radiation dose rates in a wide radioactively contaminated area, Proceedings of ICONE20, July 30-August 3, 2012, Anaheim, California, USA.

[3] D. Satoh, K. Kojima, A. Oizumi, N. Matsuda, T. Kugo, Y. Sakamoto, A. Endo and S. Okajima, Development of Calculation system for decontamination Effect, CDE, JAEA-Research 2012-020.

[4] Y. Iwamoto, D. Satoh, A. Endo, Y. Sakamoto, M. Kureta and T. Kugo, Study on Soil Decontamination and Dose Rate Reduction Effect, JAEA-Technology 2011-026.

[5] The decontamination model project accomplishment report (JAEA). 